Geophysical Research Abstracts, Vol. 9, 03822, 2007

SRef-ID: $1607-7962 /$ gra/EGU2007-A-03822

(C) European Geosciences Union 2007

\title{
Impact of different regression frameworks on the estimation of the scaling properties of radar-rainfall
}

\author{
G. Villarini (1), J.B. Lang (2), F. Lombardo (3), F. Napolitano (3), F. Russo (3) and \\ W.F. Krajewski (1)
}

(1) IIHR-Hydroscience \& Engineering, The University of Iowa, Iowa City, Iowa, USA, (2)

Department of Statistics and Actuarial Science, The University of Iowa, Iowa City, Iowa, USA,

(3) Department of Hydraulics, Highways and Roads, University of Rome "La Sapienza",

Rome, Italy

(witold-krajewski@uiowa.edu / Fax: +1 319-3355238 / Phone: +1 319-3355231)

Rainfall is characterized by high variability both in space and time. Despite continuous technological progress, there is still a lack of instruments able to span across several spatio-temporal scales. To remedy this situation, multifractal analysis of spatial precipitation has become a standard tool to study its physical nature across a wide range of scales. To estimate the scaling function, it has been common practice to use ordinary least squares (OLS) regression. Unfortunately, the validity of the OLS approach depends to some extent on core assumptions that are violated in this setting. For example, errors are likely non-normal, heteroskedastic, and/or correlated. For this reason, the authors propose the use of alternative regression approaches (bootstrapping regression, semi parametric linear model, and multilevel normal linear model) that appropriately accommodate the more complex error structure. The authors show how these different approaches exert a significant impact (comparable to the effects of ground clutter) on the multifractal analysis of radar-rainfall. Additionally, the uncertainties associated with the construction of the scaling function solely due to the regression procedure will be presented. The radar data come from the polarimetric Cband weather radar located in Rome, Italy. The scaling properties have been computed for a square domain centred on the radar site with a side length of $128 \mathrm{~km}$ and a finest resolution of $1 \mathrm{~km}^{2}$. 